\title{
'Gagner les Nations à Jésus' : Entreprises Missionnaires et Guerre Spirituelle en Afrique
}

\author{
Sandra Fancello \\ CEMAf - Paris
}

\begin{abstract}
Depuis les Indépendances africaines des années 1960, et le régime d'alliance entre des Églises « officielles » et les Partis uniques, jusqu'au nouveau régime de libéralisation religieuse des années 1990 initié par les Conférences nationales, les entreprises missionnaires, issues de l'époque coloniale, mais aussi les nouvelles Missions internationales, ont à négocier leur rapport avec le pouvoir politique. Cette contribution interroge l'évolution contemporaine de la notion "d'Église missionnaire » dans ses rapports complexes avec certaines formes de nationalisme religieux, et l'extension dans ce contexte du sens même de la « mission » comme régénération morale de la nation.
\end{abstract}

\section{Keywords}

nations, nationalismes, missions, Ghana, Côte d'Ivoire

Depuis les Indépendances africaines des années 60, et le régime d'alliance entre des Églises «officielles » et les Partis uniques, jusqu'au nouveau régime de libéralisation religieuse des années 90 initié par les Conférences nationales, les entreprises missionnaires, celles issues de l'époque coloniale comme les nouvelles Missions internationales, ont dû négocier leur rapport avec le pouvoir politique. A mi-chemin entre les Églises missionnaires coloniales et les nouveaux ministères, des Églises internationales telles que l'Église des Assemblées de Dieu, d'origine américaine ou européenne, ou la Church of Pentecost du Ghana, sont devenues, dès les années 1950, des Églises nationales et sont aujourd'hui plus que jamais des actrices des changements sociopolitiques en cours marqués par le réveil des ethno-nationalismes. Du panafricanisme de Kwame Nkrumah, qui renait entre autre dans le milieu des Églises évangéliques ghanéennes, à la politique d'ivoirisation des cultes dans les Églises de Côte-d'Ivoire, les enjeux de la gestion de ces entreprises religieuses et de leur irruption dans l'espace public interpellent tous les gouvernements.

Cette contribution interroge l'évolution contemporaine de la notion «d'Église missionnaire » dans ses rapports complexes avec certaines formes de 
nationalisme religieux, et l'extension dans ce contexte du sens même de la «mission » comme régénération morale de la nation. De la forme première et familière de l'Église des missionnaires, avec ses stations et ses oeuvres, aux nouvelles Missions ou Églises transnationales, les interférences, les télescopages et les rappels ne cessent de se multiplier. Le premier travail missionnaire a contribué à la formation d'une conscience ethnique forgée dans le moule des nations bibliques par le biais entre autres de la transcription et de la codification des langues vernaculaires (Hastings, 1997). L'enjeu politique de l'installation missionnaire se négocie alors avec les chefferies qui, après la période de méfiance ou de résistance, s'approprient les missions. Mais la stratégie d'implantation des "stations missionnaires » visait aussi, à terme, à contourner les pouvoirs locaux, en s'installant à distance (Bureau, 2002), tout en négociant avec le pouvoir colonial, notamment en faisant jouer la concurrence avec l'islam (Audoin, Deniel, 1974). Durant les mouvements d'indépendance, les élites lettrées et éveillées formées par les Églises initient ou accompagnent les mouvements d'émancipation politique et contribuent à donner à ces identités ethno-nationales une expression politique au sein des futurs États-nations. Aujourd'hui, la crise de légitimité de ces États et la faillite de la «transition démocratique » encouragent le réveil des ethno-nations, avec la complicité paradoxale des mouvances religieuses évangéliques de type pentecôtiste (religion yoruba, ivoirité, akanité, kongolité, etc.). Depuis les années 1990, les Églises historiques qui ont joué un rôle symbolique de médiation et de recours dans les conférences nationales, sont bousculées par de nouveaux ministères prophétiques qui s'appuient autant sur leurs réseaux de ressources internationaux que sur les ressorts identitaires des réveils locaux. L'entreprise missionnaire ne peut s'en tenir à une action d'évangélisation qui passe par la conversion des individus et l'éducation des populations. Elle suppose l'engagement dans une guerre spirituelle qui a une dimension mondiale et qui se donne pour objectif de « gagner la nation à Jésus ».

\section{Missions, nations et nationalismes}

Le moment historique que constitue la « rencontre coloniale » sur le continent africain dès le XIXe siècle s'est traduit, entre autre, par la formation d'Églises «missionnaires ». Leur entreprise d'indigénisation du christianisme a abouti dès le début du XXe siècle à l'émergence d'Églises africaines (AIC) par scissions ou initiatives locales, mais il est difficile avec le recul de maintenir une frontière radicale entre les premières et les secondes malgré la stigmatisation de ces initiatives comme «syncrétismes» ou «néo- 
traditionalismes». John Peel (2000) présente l'invention chrétienne de l'identité Yoruba comme une "construction interactive ", liée à la médiation des returnees du Liberia, fils d'esclaves libérés, christianisés et lettrés, transformés en agents de l'action missionnaire. Ce sont souvent les mêmes catéchistes, plus ou moins dissidents, qui participeront, à la manière du prophète Harris, en renouant avec leurs « racines » africaines, à la fondation d'Églises Indépendantes. Birgit Meyer (2002) montre de son côté l’impact de la politique culturelle des missionnaires piétistes allemands et leur contribution à l'émergence d'une «nation Éwé» (Ewe nationhood). Paradoxalement, les entreprises missionnaires d'alphabétisation et d'indigénisation du christianisme, qui ont livré les premières traductions de la Bible en langues vernaculaires, ont ouvert la voie à des formes d'appropriation des catégories chrétiennes et à l'emprunt de métaphores bibliques qui serviront de pilier à la constitution des imaginaires politiques de la nation noire, sur le modèle des tribus d'Israël. La formation d'identités ethniques à prétention nationaliste est donc en partie le produit de l'indigénisation des Églises missionnaires qui a abouti, dès la période coloniale, à la formation et à l'éveil d'identités ethnonationales souvent inédites.

L'expression Église Africaines Indépendantes (Independent African Churches) désigne au départ les Églises séparatistes fondées en Afrique, par des Africains pour des Africains dans le contexte de l'apartheid ou du régime de séparation coloniale, en rupture avec les Églises missionnaires. Mais de nouvelles générations d'Églises, qui ne sont pas issues de la rupture coloniale, émergent sur le continent africain. Elles sont appelées African Initiated Churches (équivalent des "Églises d'initiatives africaines ») ou African Instituted Churches ou encore aujourd'hui International African Churches - qui rappellent volontairement, en le détournant, le sigle AIC. Le paradoxe est que ces Églises africaines se pensent aujourd'hui à leur tour comme des «Églises missionnaires » et s'exportent hors des frontières nationales. De même que le concept de "prophétisme» n'a jamais été le propre des Églises dites «prophétiques», le concept de «mission» n'est plus le propre des Églises missionnaires, au sens historique, mais constitue une catégorie générique applicable à la plupart des entreprises religieuses transnationales. 
La Church of Pentecost du Ghana est l'un des rares cas d'Église pentecôtiste ${ }^{1}$ africaine fondée par un Blanc, le missionnaire écossais James McKeown, envoyé au Ghana en 1937 par l'Apostolic Church (Bradford). Il s'en séparera pour créer sa propre Église avec l'intention de transmettre l'héritage aux Africains (Fancello, 2007). Cette Église s'est implantée au Ghana dans la région ashanti (Asamankese, Winneba), et s'est caractérisée d'emblée par une composante identitaire ethno-nationale forte qui en demeura, après le retrait du missionnaire, un trait marquant. Malgré ses origines «missionnaire», au sens historique du terme, la Church of Pentecost se pense aujourd'hui comme une Église africaine ${ }^{2}$. Son ambition d'expansion transnationale va de pair avec la revendication du statut d'Église «indigène » et son engagement missionnaire n'entame pas l'attachement fort de cette Église à son foyer identitaire étroitement associé au groupe Ashanti du Ghana. La Church of Pentecost s'est progressivement dotée d'une mission historique qui fait du Ghana une "nation missionnaire » au même titre que le Nigeria pour les pentecôtistes Yoruba. Ainsi, les chrétiens Ashanti, qui forment l'essentiel du groupe Akan, se pensent comme le peuple élu de Dieu, idée empruntée à l'Ancien Testament. Le pentecôtisme s'est nourri du modèle judaïque des «nations » transmué en lieu de formation et d'expression d'identités ethno-nationales à vocation mondiale. L'akanité, en tant qu'expression d'un nationalisme culturel et religieux, se développe aussi dans le contexte de l'idéologie politique panafricaniste. Cette identité qui se construit dans l'aller-retour entre le Ghana et les États-Unis se nourrit d'une longue tradition d'échange entre Kwame Nkrumah, le premier président du Ghana indépendant, et la communauté noire américaine. Durant ses années de formation universitaire aux États-unis, Nkrumah fut fortement influencé par la pensée des fondateurs du mouvement afrocentriste. Mais l'expansion transnationale de la Church of Pentecost et la perception du Ghana comme «nation missionnaire » renouent également avec l’imaginaire de la suprématie du groupe Akan. Les fonctions du Ghanéen Kofi Annan (de formation méthodiste) à l'Organisation des Nations Unies sont le signe tangible, pour les pentecôtistes akan, du plan divin qui fait du Ghana le fer de lance de l'expansion missionnaire africaine.

\footnotetext{
${ }^{1}$ Au Ghana, le terme «Pentecostal » désigne les Églises pentecôtistes « classiques » telles que les Assemblées de Dieu ou l'Église de Pentecôte, et le terme "Charismatic » désigne les nouvelles Églises apparues depuis les années 1970 (Gifford, 1994 : 241), tandis qu'au Nigeria, le terme « Pentecostal » s'applique à la mouvance pentecôtiste, le terme "Charismatic » étant réservé au Renouveau catholique (Hackett, 1998 : 259).

2 Elle serait aujourd'hui la plus importante dénomination pentecôtiste du Ghana et la deuxième Église du pays après l'Église catholique (Gifford, 1998 : 76; ter Haar, 1994 : 225).
} 
Dans le contexte colonial, «l'Église» Jamaa, inscrite dans la mouvance catholique charismatique, fondée au Zaïre au début des années cinquante par le célèbre missionnaire belge Placide Tempels (Fabian, 1994), constitue un autre cas d'Église africaine fondée par un blanc mais avec un destin différent. Issu d'une dissidence avec le clergé catholique et censuré, le mouvement Jamaa fut assimilé à une secte. Accusé de soutenir la rébellion contre l'État, le mouvement fut l'objet de persécutions et le missionnaire rentra en Belgique en 1967. Mais l'Église n'en demeura pas moins présente et après l'accession au pouvoir de Mobutu, le climat religieux étant plutôt favorable aux innovations religieuses, l'Église entama un processus «d'africanisation». Tandis que des intellectuels catholiques zaïrois contribuaient à la formation de l'idéologie nationaliste et à l'élaboration de la théologie de «l'authenticité » (Fabian, 1994 : 259), l'Église Jamaa devenait l'illustration de la «philosophie bantou» et prit part à la lutte de la société pour son indépendance culturelle. D’autres Églises de type prophétique telles que l'Église du Christianisme Céleste ou l'Église kimbanguiste véhiculent l'idée d'un destin messianique des groupes ethnonationaux (Yoruba, Kongo) auxquels elles restent attachées. Pour ces chrétiens, l'identité céleste ou kimbanguiste est inséparable de leur lien avec le christianisme primitif et de leur fondation par un «Christ noir» (MokokoGampiot, 2004).

De même la notion « d'Ivoirité » en Côte-d'Ivoire, participe, pour le milieu évangélique, de la construction d'une identité chrétienne ivoirienne. Il s'agit toujours d'une identité chrétienne de type évangélique, pensée dans des termes bibliques. L'ivoirité est particulièrement représentative d'une construction identitaire nationaliste qui transcende les «ethnies», quoique étroitement associée au groupe akan (Dembélé, 2002 : 167). Il s'agit avant tout d'une identité nationale reliée aux «chrétiens du sud» en rupture avec les «musulmans du nord» qui sont aussi associés aux «étrangers» (les Dioula, mais aussi les migrants burkinabé) majoritairement musulmans. La confrontation avec l'islam, qui dans ses formes contemporaines emprunte au pentecôtisme ses méthodes de prosélytisme militant, demeure d'une pertinente actualité, notamment dans des pays tels que le Nigeria, le Niger et la Côte d'Ivoire (Miran, 2000; Marshall, 2006).

L'ivoirisation avait en réalité commencé sous le régime de Félix HouphëtBoigny par l'ivoirisation des cadres. Mais l'ivoirisation des cultes semble avoir précédé la conceptualisation progressive de l'identité nationale sous la forme de «l'ivoirité » exploitée par Henri Konan Bédié dans une phase ultérieure. Dès le début des Indépendances, de nombreux conflits liés à la succession des imams opposent imams ivoiriens et burkinabé. Plus tard, au milieu des années 1980, des musulmans du Sud ivoirien (bété, dida, adjoukrou) refusent la 
domination des «étrangers» (principalement les Dioula) dans les mosquées et revendiquent des droits liés à l'autochtonie ${ }^{3}$. Parallèlement, des pasteurs pentecôtistes burkinabé témoignent d'un processus similaire au sein des Églises type Assemblées de Dieu, dont l'implantation en Côte-d'Ivoire a suivi les chemins de la migration burkinabé. A un moment que les pasteurs interrogés situent au milieu des années 1980, ces derniers durent accepter d'être progressivement remplacés par des pasteurs ivoiriens ${ }^{4}$. Ainsi pour le pasteur Adama Ouedraogo : "l'ivoirité était dans l'Église avant d'être dans l'État! ».

\section{Des Églises nationales à vocation missionnaire}

L'engagement autant que la visibilité des Églises pentecôtistes dans le champ politique est étroitement lié aux relations entretenues entre les Églises et l'État, voire aux liens qui se tissent avec le Chef de l'État lui-même et à la nature de l'option religieuse de ce dernier. Au Ghana, les principales Églises chrétiennes ${ }^{5}$ sous contrôle de l'État, ont joué un rôle majeur dans la construction de l'État moderne, en particulier par le biais de leur écoles «missionnaires» qui ont contribué à former l'élite de ce pays depuis la fin du XIXe siècle. L'influence des Églises dans la société ghanéenne est telle que, comme le rapporte Paul Gifford : "Today it is impossible to begin or end meetings or any kind without Christian prayer (in which Muslims seem ready to participate)》 (2004 : 21). Les relations entre les Églises pentecôtistes et les gouvernements ghanéens successifs varient sensiblement d'un chef d'État à un autre. Globalement, les Églises chrétiennes au Ghana bénéficient d'une certaine liberté religieuse et il n'y eut pas au Ghana d'interdiction des Églises, comme au Togo ou dans d'autres pays africains dans le cadre des partis uniques. Au contraire, les Églises chrétiennes au Ghana sont partie prenante dans le développement du pays, ainsi que dans la promotion de la culture traditionnelle. Elles jouèrent déjà un rôle important dans le programme de développement de l'éducation et de la santé lancé par le président Nkrumah dès le début des années cinquante ${ }^{6}$. Mais après la chute de

\footnotetext{
${ }^{3}$ Marie Miran, communication personnelle .

4 Enquêtes personnelles à Ouagadougou et Bobo-Dioulasso en 2005 et 2006 auprès de pasteurs burkinabés rapatriés, ainsi qu’à Abidjan en juin 2007.

5 Parmi les principales Églises influentes dans le pays on compte l'Église catholique, et parmi les Églises protestantes, l'Église méthodiste, l'Église presbytérienne, l'Église anglicane et plusieurs Églises pentecôtistes telles que l'Apostolic Church, la Church of Pentecost, la Christ Apostolic Church et les Assemblies of God.

${ }^{6}$ Dans le contexte « revivaliste » protestant, le " charismatique » Nkrumah n'hésitait pas à « exprimer son socialisme panafricain à l'aide de métaphores chrétiennes » (Gifford, 1998 : 58),
} 
Nkrumah, l'arrivée au pouvoir du capitaine Rawlings en 1981, après un premier coup d'État manqué en 1979, est perçue comme une «seconde venue» ("Second Coming») du leader, tandis que ses initiales J. J. sont métaphoriquement traduites par la presse par "Junior Jesus» ou, dans l'opposition, «Junior Judas» (Gifford, 1998 : 62).

En janvier 1994, les Églises protestantes, catholique, pentecôtistes et indépendantes participaient à la célébration commémorant l'anniversaire de la IVe République. Au cours du service, seul le leader pentecôtiste DuncanWilliams se démarqua par son discours : Th ere is an invisible hand over the nation bolding back bloodshed (...) Pray that this invisible hand may remain on our leaderss, établissant ainsi un lien direct entre le président Rawlings, la relative stabilité du pays et la grâce divine. La presse "gouvernementale », en l'occurrence le Daily Graphic, accorda davantage d'importance à l'intervention du leader pentecôtiste qu'aux autres orateurs. Par ailleurs, contrairement à la posture de la plupart des Églises chrétiennes qui considèrent que les chrétiens ne sont pas concernés par la politique (c'est l'une des limites du «retrait du monde»), l'évangéliste Owusu Tabiri, un autre leader pentecôtiste très populaire au Ghana, dissident de la Church of Pentecost, s'exprime, en 1995, dans le sens d'une plus grande implication des Églises et des leaders religieux dans la sphère politique :" Ghana will not experience the Glory of God until the leader becomes a Christian, déclare-t-il, I have asked the Lord to intervene to give us [in the next election] his own choice » (Gifford, 1998 : 86). Outre la communauté de fidèles qu'elle représente, la Church of Pentecost, aujourd'hui la première Église pentecôtiste au Ghana, est considérée comme une Église nationale. Son président, l'Apôtre M. K. Ntumy est également l'actuel président du Ghana Pentecostal Council. Comme beaucoup de leaders religieux, il prend par ailleurs grand soin de ménager ses relations avec l'actuel chef de l'État (Kufuor, qui est catholique), pourvoyeur de moyens à l'occasion, afin de garantir à l'Église une forme de reconnaissance, voire de caution et de protection politique.

Mais les Églises ghanéennes ont aussi produit quelques uns des évangélistes africains les plus célèbres, tels que Nicholas Duncan-Williams, Mensa Otabil, Charles Agyin Asare ou Owusu Tabiri, et donc ouvert la voie à des entreprises missionnaires d'un nouveau type. Au delà des relations de convenance et de bonne entente, ces leaders sont les agents militants d'une « nation missionnaire » qui ferait du Ghana une nation chrétienne exemplaire, portée par un peuple élu entre tous, élevée au dessus du continent africain et

de même que les discours du Convention People's Party (CCP) se teintaient de connotations chrétiennes. 
de ses pesanteurs historiques, afin d'engager la ré-évangélisation du monde. L'expansion fulgurante de la Church of Pentecost, présente dans près de soixante pays de mission, est, pour ses leaders, la confirmation de ce plan divin. Mais dans ses implantations missionnaires, hors du Ghana, la Church of Pentecost, perçue comme une Église « étrangère » essentiellement vouée à l'encadrement des communautés de migrants ghanéens - notamment par l'usage systématique de la langue twi des Ashanti comme langue liturgique -, a du souvent faire face aux réveils nationalistes conduits en général par les agents «autochtones » qu'elle avait formés. La Côte-d'Ivoire et le Togo, deux pays frontaliers du Ghana qui furent les relais francophones les plus importants de la Church of Pentecost jusque dans les années 1990, se présentent comme les deux cas les plus représentatifs de la dimension politique des conflits religieux. Ainsi, au début des années 1980, un jeune leader ivoirien contestant la présence de missionnaires ghanéens au sein de l'Église de Pentecôte de Côted'Ivoire, milite pour l'autonomie de l'Église nationale vis-à-vis du siège ghanéen, pour l'adoption de la langue française dans les assemblées et pour la nomination d'un président ivoirien à la tête de l'Église. Dans le conflit ouvert qui s'engage avec les dirigeants, notamment à travers la presse ivoirienne, le dissident Yao Bio se proclame président de l'Église de Pentecôte de Côted'Ivoire, usurpant ainsi la dénomination de l'Église ghanéenne qui l'attaque en justice. Mais au terme de cette bataille juridique qui dure jusqu'en 2001, le dissident nationaliste obtient les faveurs de la presse, voire du gouvernement par le biais du Ministère de l'Intérieur - qui reconnaît la légalité de l'Église de Yao Bio et condamne l'Église ghanéenne à modifier sa dénomination ${ }^{7}$ afin de la distinguer de l'Église "ivoirienne ${ }^{8}$. Dans le contexte idéologique de l'ivoirité et dans la continuité de l'ivoirisation des cultes, la préférence nationale s'est donc exercée en faveur du dissident ivoirien en dépit des arguments juridiques avancés par les leaders ghanéens.

$\mathrm{Au}$ Togo, après une période d'interdiction qui contraint la jeune Église missionnaire à la clandestinité ${ }^{9}$, les missionnaires ghanéens sont confrontés,

\footnotetext{
${ }^{7}$ En dépit du fait que celle-ci disposait d'un arrêté ministériel délivré par le même Ministère de l'Intérieur en 1966 attestant de l'enregistrement de l'Église de Pentecôte par les missionnaires ghanéens.

8 Depuis 2003, l'Église ghanéenne a opté pour la dénomination «Église de Pentecôte Internationale ».

${ }_{9} \mathrm{Au}$ Togo, l'Église de Pentecôte fut durant plusieurs années sous le coup de l'interdiction des Églises assimilées à des «sectes» de 1978 à 1984. La violence de la répression poussa les pasteurs à formuler un recours auprès du Chef de l'État, au cours de l'année 1984, lequel leur suggéra de se placer sous la conduite de l'Église Évangélique du Togo (aujourd'hui Église Évangélique Presbytérienne du Togo). Loin de se faire dans la clandestinité, cet accord dit
} 
dès la fin des années 1980, aux pressions nationalistes de leurs auxiliaires togolais qui se voient refuser le statut d'apôtre et confinés à un statut subordonné. Là encore, la fermeté de l'Église centrale, contrôlée par une élite de pasteurs et apôtres exclusivement ashanti, aboutit à la scission de l'Église de Pentecôte du Togo dont les pasteurs togolais prennent le contrôle et la propriété. Le conflit qui opposa les deux Églises de la même dénomination fit l'objet d'une décision de justice qui a contraint l'Église (ghanéenne) à changer de nom. La préférence nationale des États africains dans le champ religieux a poussé les autorités ivoiriennes et togolaises à soutenir les revendications nationalistes des dissidents, leur accordant la légitimité religieuse et la propriété des biens acquis durant l'affiliation avec la Church of Pentecost du Ghana. Affaiblie par les échecs de l'impérialisme akan à l'épreuve de l'ivoirité et autres nationalismes, l'Église missionnaire, renonçant à s'afficher trop ouvertement dans l'espace public, entend s'imposer autrement et opte, à la fin des années 1990, pour la «nationalisation» progressive des dirigeants nationaux et le retrait progressif des leaders ghanéens dans les «pays missionnaires » afin d'accélérer l'autonomie des Églises nationales, notamment francophones, et de leur permettre une meilleure intégration dans le paysage religieux national. Ainsi au Burkina Faso, l'adhésion de l'Église de Pentecôte à la Fédération des Églises et Missions Évangéliques (FEME ${ }^{10}$ ) qui se pense comme une institution profondément nationale, fut longtemps différée et ne fut rendue possible qu'au retrait du dernier missionnaire ghanéen en 1999, près de quarante ans après l'implantation de l'Église dans ce pays. La «nationalisation » des dirigeants a permis à l'Église de Pentecôte de hisser ses représentants au plus près de la sphère politique, comme en témoigne l'élection de l'apôtre Sidiki Traoré ${ }^{11}$, à la vice-présidence du Conseil national des Églises Protestantes et Évangéliques de Côte-d'Ivoire $\left(\mathrm{CNEPE}-\mathrm{CI}^{12}\right)$ en 2003. La photographie de l'apôtre serrant la main du président L. Gbagbo fut publiée dans la revue de l'Église (Pentecost Fire), attestant le retour de l’Église ghanéenne dans les bonnes grâces du gouvernement.

«d'intégration » fit l'objet d'une demande auprès du Ministère de l'Intérieur qui donna son accord. Au début des années 1990, lorsque fut entamé le " processus de démocratisation », restaurant, entre autre, la liberté de culte, l'Église de Pentecôte retrouva sa dénomination.

${ }^{10}$ Créée en 1961, dans la continuité de la Fédération des Églises et Missions Protestantes de l'AOF dissoute à la suite des indépendances, la FEME rassemble aujourd'hui une douzaine d'Églises.

11 Président de l'Église de Pentecôte de Côte-d'Ivoire et membre du Conseil Exécutif International de la Church of Pentecost au sein duquel il représente la « zone francophone ».

${ }^{12}$ Qui remplace la Fédération des Églises de Côte-d'Ivoire (FECI). 


\section{Prosélytisme missionnaire et "spiritualité politique "}

Au regard des réussites et des impasses de ces Églises nationales, à vocation missionnaire, il faut prendre en compte le travail parallèle des Ministères transnationaux et de leurs leaders charismatiques. Le continent africain est, dès les années 1960, le terrain d'action de plusieurs personnages charismatiques transnationaux tels que Reinhard Bonnke ou Yonggi Cho dont les croisades d'évangélisation sillonnent l'Afrique contemporaine (Corten, Marshall, 2001). La force de la mouvance pentecôtiste repose à la fois sur un réseau de communautés de base, des "Églises de maison », et sur le rassemblement des foules dans les stades ou lieux collectifs notamment lors des campagnes d'évangélisation. Mais ce qui frappe également l'observateur, c'est la stratégie de gestion de la politique "par le haut », de contrôle et de séduction des chefs d'États. Ainsi, au Bénin, « les 'grands' pasteurs se revendiquent pasteur de tel ou tel chef d'État, ce qui renforce leur légitimité religieuse, notamment au niveau international » (Strandsbjerg, 2005). Certains évangélistes passent directement par le président, comme Reinhard Bonnke, qui fut reçu par le président Compaoré à Ouagadougou (1990) et le président Moi à Nairobi (1991), tandis qu'en 2001, Laurent Gbagbo recevait le Coréen Yonggi Cho à Abidjan. Si les Églises établies sont plus discrètes, le rôle des Ministères prophétiques et des pasteurs conseillers dans la presse, les médias et l'opinion, est plus actif. De la même manière que les missionnaires négociaient avec les chefferies, ces «ministères» convoitent la caution du «haut» et réciproquement.

Pour les nouvelles Églises, la sphère politique africaine actuelle est souvent perçue comme l'instrument du Diable (Ellis, Ter Haar, 1998 : 177) et l'imaginaire politique est alors associé aux "forces invisibles ", des catégories du mal assimilées aux « forces occultes » (De Surgy, 2000 : 50). Le rapport au politique passe par la "guerre spirituelle » contre les démons qui mènent le monde. La diabolisation de la sphère politique est un trait commun aux Églises pentecôtistes de nombreux pays d'Afrique, comme le rapporte Yan Droz à propos du Kenya où «l'on raconte que le culte à Satan est largement répandu parmi les proches du gouvernement kenyan (...) il serait la raison de son maintien au pouvoir en dépit de l'opposition de la majorité de la population » (Droz, 1997 : 102). Ce discours est largement véhiculé par le biais de rumeurs qui auraient accompagné le processus de démocratisation du pays. Chaque pays offre, selon son contexte politique récent, des possibilités d'interprétation et de légitimation aux dirigeants pentecôtistes. Ainsi, lors d'une croisade d'évangélisation à Ouagadougou (Burkina Faso) en février 1999, les dirigeants 
ghanéens de la Church of Pentecost ont saisi l'occasion que leur offraient la campagne et les élections présidentielles organisées quelques mois auparavant pour dénoncer le Président de l'État, et l'assimiler, de manière détournée mais en termes clairs, au Démon:

«Voilà ce que Jésus a dit : 'l'ennemi, le voleur n'est venu que pour voler, détruire et tuer' (. . .) je crois que l'année passée il y a eu des élections dans ce pays $^{13}$ (. . .) Je voudrais vous dire que la personne qui est en train de vous tromper aujourd'hui c'est le diable et il vous trompe. Le diable est venu pour tuer premièrement, voler et détruire. (. . .) La plupart des gens veulent voter. Pourquoi voulez-vous voter pour Satan ? Les gens veulent voter pour Satan alors que son intention est très claire : il est venu tuer, voler et détruire. »

La diabolisation de la sphère politique assimile donc clairement, pour certains pasteurs, le vote politique à un acte satanique. Voter, c'est-à-dire participer à l'activité et aux enjeux politiques, c'est négocier avec Satan et s'investir dans la vie politique, c'est contribuer à l'instauration de l'empire de Satan. Outre l'impact de la métaphore, les pasteurs touchent là à un point sensible commun à de nombreux pays africains ou latino-américains qui souffrent du désengagement et du manque de moyens de l'État. Mais on peut aussi « voter pour Jésus », comme dans d'autres pays africains, tel que le Bénin de Kérékou II, où le président a pu mettre en avant sa conversion pentecôtiste lors de sa seconde élection (" une Bible pour chaque Béninois») et en fait un atout de sa réussite politique. La sphère politique est alors surinvestie par les chrétiens évangéliques, même si, dans ses "imaginaires politiques », le pentecôtisme «récupère, amalgame les imaginaires disponibles et ses 'traductions' relèvent plutôt de 'mésinterprétations constructives' (working misunderstanding)» (Corten, Mary, 2000 : 17). La notion de «spiritualité politique ", inspirée de la gouvernementalité d'inspiration foucaldienne, telle qu'elle est utilisée par Ruth Marshall dans le contexte nigérian, aboutit à l'extension de la «culture pentecôtiste » à l'ensemble de l'espace social et politique:" Popular culture and the media have been colonised by Pentecostal images and discourse, revealing that its effects are by no means limited to the sphere of religious practice » (Marshall, 2006 : 5). Au Nigeria, comme dans bien d'autres pays, la "pentecôtisation » du discours politique n'est pas sans rapport avec le dynamisme musulman qui constitue, aux yeux de ces chrétiens, une menace non pas seulement d'ordre religieux, mais bien politique.

Dans un autre contexte, l'Église de Pentecôte de Côte-d'Ivoire a pu prendre part à un « sommet de prière » (2001) destiné à asseoir le pouvoir du

${ }^{13}$ Nous sommes en février 1999, Blaise Compaoré vient d'être réélu en novembre 1998. 
président Laurent Gbagbo face à la "menace musulmane » que représente l'expansion de l'islam dans ce pays. A cette occasion, plusieurs pasteurs ont mis en avant l'élection historique du premier " président chrétien $»^{14}$ à la tête du pays, preuve que la «main de Dieu est sur la Côte-d'Ivoire». Les prières successives de ces leaders visaient toutes à protéger les membres du gouvernement de «l'assaut des ennemis », qui dans l'imaginaire politique de ce pays désignent les musulmans du nord, et à invoquer la puissance divine pour guider les leaders politiques, afin " qu'ils ne chutent pas ", en négociant par exemple avec l'adversaire politique désigné, Alassane Ouattara. La sphère politique se trouve ainsi enveloppée, dans la perception pentecôtiste, par la «main de Dieu » qui protège la « nation chrétienne » contre ses ennemis. Cette représentation de la sphère politique comme un lieu à protéger, voire à libérer de ses assaillants (la question de la candidature de Alassane Ouattara à l'élection présidentielle est dans tous les esprits), par la puissance de la prière, est une dimension nouvelle $\mathrm{du}$ discours pentecôtiste qui quitte momentanément le jeu de l'individualisation pour s'adresser à la « nation » et au gouvernement («Côte-d'Ivoire, lève-toi! »), voire à Dieu lui-même (« Dieu, délivre la Côte-d'Ivoire!»). Comme l'explique J.-P. Bastian, "Cette dimension géopolitique de la mobilisation des forces de l'invisible caractérise un discours qui ne s'adresse plus seulement à l'individu, mais à l'État-nation qu'il faut donc libérer» (Bastian, $2000: 219)$.

Ainsi, l'investissement progressif des Églises dans la sphère politique, comme l'illustre la participation des Assemblées de Dieu du Burkina Faso au processus électoral (Laurent, 2000) ou l'Église de Pentecôte de Côte-d'Ivoire, vise à placer l'avenir politique du pays dans «la main de Dieu », au point que «le discours évangélique semble s’imposer comme une nouvelle idéologie politique en Afrique après la fin des régimes d'orientation marxiste» (Strandsbjerg, 2005 : 225). Par contraste, les nouveaux gouvernements africains formés par des leaders évangéliques sont fortement soutenus par les Églises nationales qui organisent régulièrement des « sommets de prière » ou des «Conférences de leaders » en faveur des responsables politiques (Gifford, 1998; Laurent, 2000; Strandsbjerg, 2005; Fancello, 2006). De même, « les services religieux ou les croisades sont l'occasion de réaffirmer la nécessité d'obéir aux autorités politiques, car leur mission est légitimée par la volonté divine » (Droz, 2000 : 96). Lors du « Forum des religions » organisé en 2001, «le rôle des Églises dans cette politique de réconciliation et de pardon a d'emblée été considéré par tous comme essentiel, et les représentants des

\footnotetext{
14 Par « chrétien », il faut entendre « évangélique », ces pasteurs ne considérant pas l'ancien président Houphouët-Boigny, catholique, comme un « vrai chrétien ».
} 
Églises ont été des acteurs privilégiés autant que des médiateurs recherchés » (Mary, 2002 : 83). Dans le conflit qui divise le pays depuis plus de quatre ans, le mouvement ultranationaliste des « jeunes patriotes », partisans du président Laurent Gbagbo, s'est invité dans le débat politique national. Aujourd'hui Richard Banégas rapporte qu'au sein du «Parlement de Yopougon », l'un des espaces de « débat démocratique » apparus dans tout le pays depuis le début de la crise, «on invite la plupart du temps un pasteur d'une église évangélique (...) qui tente de captiver l'attention des passants par des harangues où la liturgie pentecôtiste de la délivrance tient souvent bonne place» (2006 : 144). La délivrance des démons prend clairement une dimension politique.

\section{Mission évangélique et prosélytisme humanitaire}

Parallèlement à la guerre spirituelle, il y a aussi place, comme dans les mouvements islamiques, pour l'action humanitaire. Dans la continuité de la tradition missionnaire d'éducation et de soins, les Églises pentecôtistes africaines se sont engagées dans des oeuvres d'utilité publique, construisant écoles et centres de soins. Cette nouvelle forme d'institution et d'action qui associe prosélytisme et action humanitaire s'apparente parfois à un mode de redistribution tacite des sommes prélevées sur la dîme et les offrandes. Par la mise en évidence des moyens dont elles disposent, les Églises donnent implicitement une idée de leur puissance financière mais témoignent aussi de leur vocation à être des associations religieuses et sociales à but non lucratif. Comme l'affiche l'Église de Pentecôte sur son site Internet : «Th e Church of Pentecost, is a worldwide, non-profit-making Pentecostal church ${ }^{15}$. L'investissement de l'Église dans les services sociaux se traduit par la création en 1979 de la « Pentecost Social Welfare Association »(PENTWAS) qui devient PENTSOS (Pentecost Social Services) en 1983. Ayant acquis depuis 1992 le statut d'Organisation non gouvernementale, PENTSOS est le corps par lequel la Church of Pentecost entend contribuer au développement socio-économique du Ghana. Elle est enregistrée auprès de la Ghana Association of Private Voluntary Organisations in Development (GAVPOD), une organisation créée en 1980 et qui chapeaute les ONG ghanéennes et donne à leurs projets " une surface économique et financière » (Fath, 2003 : 2) par le biais d'association avec d'autres ONG, notamment américaines, comme CARE International, dans la lutte contre le sida. La création d'un service social et son accès au statut d'ONG ne constituent pas une entreprise distincte, en marge des activités de

15 http://www1.thechurchofpentecost.com/church/mission.asp 
l'Église. Elle participe au contraire, au même titre que les principaux moteurs d'évangélisation, à l'avènement d'une société chrétienne régie en tout point par les principes bibliques. La création d'une organisation aujourd'hui quasi autonome engagée pour le développement socio-économique de son pays est le fruit d'une initiative récente au sein de la Church of Pentecost qui intègre et s'inspire de la montée en puissance des organisations non gouvernementales à caractère confessionnel, notamment de culture évangélique, tel que le modèle américain peut en produire. L'aspect militant, voire offensif, s'inscrit dans la guerre déclarée contre le mal, sur les terrains de l'analphabétisme, de la maladie et de la pauvreté, qui ne sont que les manifestations de Satan sur la terre, ses «petites victoires » temporaires. L'engagement de l'Église à travers cette organisation s'intègre néanmoins dans la continuité d'une culture religieuse qui, comme dirait $\mathrm{S}$. Fath à propos des ONG américaines, " insiste particulièrement sur la norme biblique, la conversion individuelle et l'engagement. Conversionnistes et militantes, [ces organisations] posent explicitement comme fondement de leur action la référence au message de Jésus-Christ » (2003). Au Burkina Faso, l'Église des Assemblées de Dieu est parmi les plus actives dans le développement rural et agricole, comme l'illustre la fédération $W e ̂ n d-Y a m^{16}$, fondée par un pasteur protestant dans la province de l'Oubritênga (Laurent, 1998). Les principales Églises du pays, regroupées sous l'égide de l'Office de Développement des Églises (ODE) et de la Fédération des Églises et Missions Évangéliques (FEME) sont engagées dans ce « développement participatif » (Yaro, 1996; Otayek, Diallo, 1998) qui ouvre la voie au développement (kyëg taoré, lit. l'avancement) du village grâce à une redéfinition du lien social et par l'organisation du travail communautaire. Les ONG constituent le fer de lance de l'action missionnaire et le creuset de ses réseaux transnationaux.

\section{Conclusion}

Pour Paul Gifford (1998; 2000), les Églises de la mouvance pentecôtiste, en investissant l'espace public, contribuent à forger une société civile et s'inscrivent ainsi dans la continuité du processus de démocratisation, même si ce processus ne va pas sans une forme d'instrumentalisation du champ politique. Cependant, cet espace public n'est pas pour autant perçu par ces chrétiens comme le lieu de l'affirmation citoyenne mais comme le lieu d'un

16 Dans la langue möré des Mossi, Wênd-Yam signifie « la volonté de Dieu ». 
combat spirituel. Ces Églises s'engagent dans une mission de "moralisation » de l'espace public ou, plus globalement, de « régénération morale » de la société, qui passe souvent par la réaffirmation des valeurs traditionnelles. Face à la défaillance des États-nation, au regard des politiques de santé et de sécurité, et de leur ressources en matière d'identité collective, les réseaux religieux, entreprises ou ministères, constituent sans doute aujourd'hui la seule alternative de recours pour les sujets. Cette lecture «progressiste » méconnait les ressorts identitaires des ethno-nationalismes ou des «patriotismes » dans lesquels puisent certaines entreprises missionnaires. Le message qui veut que «Dieu est américain » (Colosimo, 2006) se décline, sur le terrain africain, en «Dieu est ivoirien » ou « Dieu est ghanéen ». En un mot, de l'époque coloniale au monde contemporain, l'idée de nation religieuse reste un des ressorts majeurs de l'action missionnaire.

\section{Bibliographie}

Audoin J., Deniel R., 1974, L'islam en Haute-Volta à l'époque coloniale, Paris, L'Harmattan, INADES.

Banégas R., 2006, «La France et l'ONU devant le 'parlement' de Yopougon : paroles de 'jeunes patriotes' et régimes de vérité à Abidjan », Politique africaine, no 104, pp. 141-158.

Bastian J.-P., 2000, «Imaginaire pentecôtiste et confessionalisation de la politique au Costa Rica » in Corten A. \& Mary A. (éds.), Imaginaires politiques et pentecôtismes (Afrique/ Amérique latine), Paris, Karthala, pp. 213-232.

Bureau R., 2002, "Flux et reflux de la christianisation camerounaise » in Bureau R. (éd.), Anthropologie, religions africaines et christianisme, Paris, Karthala, pp. 339-358.

Colosimo J.-F., 2006, Dieu est américain. De la théodémocratie aux États-Unis, Paris, Fayard. Comaroff J. \& J. 1991, Of Revelation and Revolution Christianity, Colonialism and Consciousness in South Africa, vol. 1., Chicago, University of Chicago Press.

Dembélé O., 2002, « La construction économique et politique de la catégorie 'étranger' en Côte-d'Ivoire » in Le Pape M. Vidal C. (éds.), Côte-d'Ivoire, l'année terrible (1999-2000), Paris, Karthala « Les Afriques », pp. 122-171.

De Surgy A., 2000, "Le choix du monde spirituel comme espace public » in Corten A. \& Mary A. (éds.), Imaginaires politiques et pentecôtismes (Afrique/Amérique latine), Paris, Karthala, pp. 41-59. 
Fabian J.,1994, "Jamaa : A Charismatic Movement Revisited », in Blakely T. D., van Beek W. E. A. \& Thomson D. L. (eds.), Religion in Africa. Experience \& Expression, London, James Currey, pp. 257-274.

Droz Y., 2000, "Les origines vernaculaires du Réveil pentecôtiste kényan. Mobilité sociale et politique » in Corten A. \& Mary A. (éds.), Imaginaires politiques et pentecôtismes (Afrique/ Amérique latine), Paris, Karthala, pp. 81-101.

Fancello S., 2006, "Akanité et pentecôtisme : identité ethno-nationale et religion globale ", Autrepart, "La globalisation de l'ethnicité?", no 38/2 pp. 81-98 .

2006, Les aventuriers du pentecôtisme ghanéen. Nation, conversion et délivrance en Afrique de l'Ouest, Paris, ird-karthala.

2007, "Le plan de Dieu pour le Ghana : une 'Église en héritage' », Afrique \& bistoire, no 7, pp.171-195.

Hastings Adrian, 1997, The Construction of Nationhood, Ethnicity, Religion and Nationalism, Cambridge University Press.

Fath S., 2004, «Les ONG évangéliques américaines », Sciences bumaines, no 155.

Fourchard L., 2005, « Le Nigeria, une nation missionnaire? » in Fourchard L. Mary A. Otayek R. (éds.), Entreprises religieuses transnationales en Afrique de l'Ouest, Paris, Ibadan, IFRA-Karthala, pp. 333-340.

Laurent P.-J., 1998, Une association de développement en pays mossi. Le don comme ruse, Paris, Karthala.

_ 2000, «Diabolisation de l'autre et ruses de l'Esprit : les Assemblées de Dieu du Burkina Faso », in Corten A. \& Mary A. (éds.), Imaginaires politiques et pentecôtismes (Afrique/ Amérique latine), Paris, Karthala, pp. 61-79.

Mokoko-Gampiot A., 2004, Kimbanguisme et identité noire, Paris, L'Harmattan.

Gifford P., 1998, African Christianity. Its Public Role, Hurst \& Company, London.

2000, « Pentecostalism in Museveni's Uganda » in Corten A. \& Mary A. (éds.), Imaginaires politiques et pentecôtismes (Afriquel Amérique latine), Paris, Karthala, pp. 103-118.

2004, Ghana's New Christianity. Pentecostalism in a Globalizing African Economy, Bloomington \& Indianapolis, Indiana University Press.

Marshall R. A., 2006, Political Spiritualities : The Explosion of Pentecostalism in Nigeria, PhD. London.

Mary A., 2002, « Prophètes pasteurs. La politique de la délivrance en Côte d'Ivoire », Politique africaine, no 87, pp. 69-94. 
Memel-Fotê H., 1999, "Un mythe politique des Akan en Côte-d'Ivoire : le sens de l'État » in Valsecchi P., Viti F. (éds.), Mondes Akan. Identité et pouvoir en Afrique occidentale, Paris, L'Harmattan, pp. 21-42.

Meyer B., 2002, "Christianity and the Ewe Nation : German Pietist Missionaries, Ewe Converts and the Politics of Culture », Journal of Religion in Africa, 32.2, pp. 167-199.

Miran M., 2000, «Vers un nouveau prosélytisme islamique en Côte-d'Ivoire : une révolution discrète ", Autrepart, no 16, pp. 139-160.

Otayek R., Dialo D., 1998, « Dynamisme protestant, développement participatif et démocratie locale. Le cas de l'Office de développement des Églises évangéliques (Burkina Faso) », Afrique contemporaine, no 185, p. 1934.

Peel J. D. Y., 2000, Religious Encounter and the Making of the Yoruba, Bloomington (In.), Indiana University Press.

Strandsbjerg C., 2005, «Les nouveaux réseaux évangéliques et l’État : le cas du Bénin », in Fourchard L., Mary A. \& Otayek R. (éds.), Entreprises religieuses transnationales en Afrique de l'Ouest, IFRA-Karthala, pp. 223-241.

Tonda J., 1997, « De l'exorcisme comme mode de démocratisation : Églises et mouvements religieux au Congo de 1990 à 1994 », in Constantin F. \& Coulon C. (éds.), Religion et transition démocratique en Afrique, Paris, Karthala, pp. 259-284.

Yaro J. G., 1996, « De l'évangélisation au développement local : spécificités des ONG d'inspiration protestante au Burkina Faso », Colloque international ONG et développement : Du nord au sud, Bordeaux-Talence, CNRSORSTOM, Regards, pp. 277-293. 